\title{
The use of anterolateral bowing of the lower leg in the diagnostic criteria for neurofibromatosis type 1
}

David A. Stevenson, $M D^{1,4}$, David H. Viskochil, $M D, P h D^{1,4}$, Elizabeth K. Schorry, $M D^{5}$, Alvin H. Crawford, $M D^{6}$, Jacques D'Astous, $M D^{2,4}$, Kathleen A. Murray, $M D^{3,4}$, J.M. Friedman, $M D, P h D^{7}$, Linlea Armstrong, $M D^{7}$, and John C. Carey, MD, MPH ${ }^{1,4}$

\begin{abstract}
Neurofibromatosis type 1 is diagnosed clinically based on the presence of two of seven criteria developed by a panel of experts in 1987. The sixth criterion focuses on skeletal findings and is as follows: "A distinctive osseous lesion such as sphenoid dysplasia or thinning of long bone cortex, with or without pseudarthrosis." The wording for this criterion is misleading. In particular, "thinning of long bone cortex" is not the characteristic radiographic presentation, and no mention of long bone bowing is included. The distinctive clinical feature of long bone dysplasia in neurofibromatosis type 1 is anterolateral bowing of the lower leg (portion of the body delimited by the knee and ankle). The usual radiographic findings of long bone dysplasia in neurofibromatosis type 1 at first presentation, prior to fracture, are anterolateral bowing with medullary canal narrowing and cortical thickening at the apex of the bowing. We suggest that anterolateral bowing of the lower leg, with or without fracture or pseudarthrosis, is a more appropriate description of the primary finding that a clinician will use to fulfill the sixth diagnostic criterion for neurofibromatosis type 1. Clarification of this diagnostic criterion is important for the clinician and for research protocols. Appropriate interpretation will improve understanding of the natural history and pathophysiology of neurofibromatosis type 1. Genet Med 2007:9(7):409-412.
\end{abstract}

Key Words: neurofibromatosis type 1, tibial pseudarthrosis, diagnostic criteria, long bone dysplasia, tibial bowing

Diagnostic criteria for neurofibromatosis type 1 (NF1) were established by a panel of experts at a National Institutes of Health (NIH) Consensus Development Conference in 1987.1 A total of seven criteria were developed, and an individual must satisfy two of the seven criteria to fulfill the diagnosis of NF1. In 1997, members of the National Neurofibromatosis Foundation Clinical Care Advisory Board reviewed the available information and recommended a more comprehensive approach to the diagnosis and treatment of affected individuals, but no change in the NIH Diagnostic Criteria for NF1 was thought to be necessary at that time. ${ }^{2}$

The sixth criterion focuses on the distinctive skeletal findings of NF1. This criterion was stated as follows: "A distinctive osseous lesion such as sphenoid dysplasia or thinning of long bone cortex, with or without pseudarthrosis." ${ }^{1}$ Distinctive osseous lesions are uncommon in individuals with NF1 (3-5\%), ${ }^{3}$ and when this criterion was first established, in-depth knowl-

From the ${ }^{1}$ Division of Medical Genetics, Department of Pediatrics, ${ }^{2}$ Departments of Orthopedics and ${ }^{3}$ Radiology, University of Utah, Salt Lake City, Utah; ${ }^{4}$ Shriners Hospital for Children Intermountain, Salt Lake City, Utah; ${ }^{5}$ Human Genetics Division, ${ }^{6}$ Department of Orthopedics, Cincinnati Children's Hospital Medical Center, Cincinnati, Ohio; ${ }^{7}$ Department of Medical Genetics, University of British Columbia, Vancouver, British Columbia, Canada. Disclosure: The authors declare no conflict of interest.

David A. Stevenson, MD, University of Utah Health Sciences Center, Department of Pediatrics, Division of Medical Genetics, 2C412 SOM, Salt Lake City, UT 84132.

Submitted for publication March 12, 2007.

Accepted for publication April 1, 2007.

DOI: 10.1097/GIM.0b013e3180986e05 edge of this manifestation was lacking. There is no doubt that a distinctive osseous lesion is an important diagnostic criterion in some individuals with NF1, but the standard statement of this criterion is misleading. Additionally, the example of "thinning of long bone cortex" is confusing because this is an unusual presentation of long bone dysplasia, especially in young children with NF1. As a consequence, some physicians have obtained radiographs of the legs, looking for the described "thinning of the cortex" in children whose physical examination does not show any focal skeletal abnormalities. Other physicians have raised concerns about how to quantify the "thin cortex" to fulfill this diagnostic criterion. Clearly, a better description of what constitutes a distinctive osseous lesion in NF1 is needed to use this sixth criterion more effectively.

Skeletal abnormalities associated with NF1 include scoliosis, sphenoid wing dysplasia, long bone dysplasia, bone cysts, and shorter than expected stature for familial background. ${ }^{4,5}$ Probably the most characteristic skeletal abnormality observed in young children with NF1 is long bone dysplasia. The usual clinical presentation in infancy or early childhood is anterolateral bowing of the tibia, with the apex near the junction of the middle and distal thirds of the tibia (Fig. 1). In some cases, pathologic fracture occurs in the bowed region, and these fractures often do not heal normally, leading to nonunion or pseudarthrosis. ${ }^{4-6}$ The terminology used to describe the clinical and radiographic findings is often confusing, and the skeletal abnormalities exist along a continuum. Most commonly, the characteristic long bone dysplasia of NF1 is incorrectly de- 

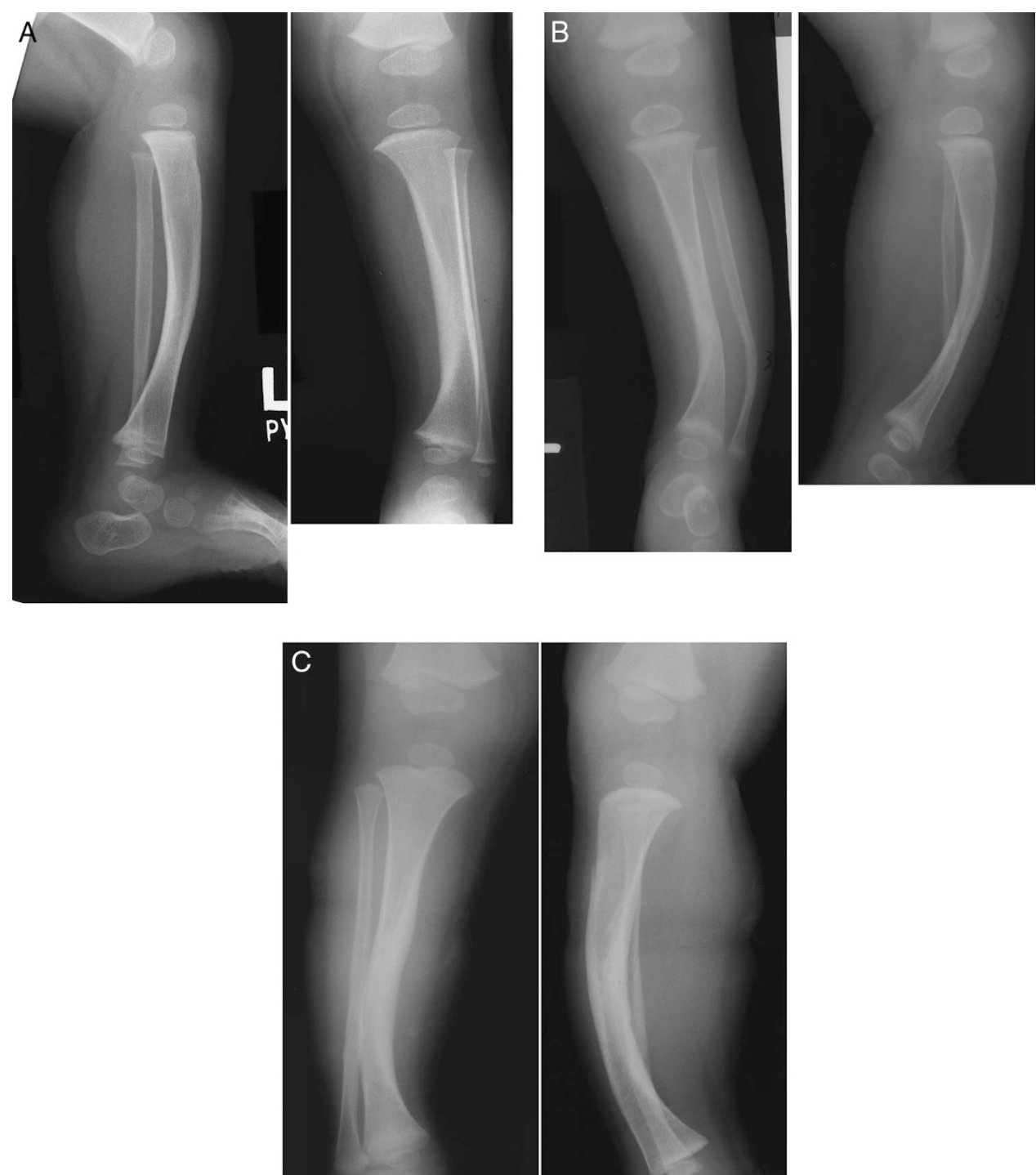

Fig. 1. Radiographs of the lower leg (both anteroposterior and lateral views) demonstrating anterolateral bowing of the tibia with cortical thickening $(A-C)$.

scribed as "congenital tibial pseudarthrosis," although the process often involves the ipsilateral fibula as well and may affect the radius and/or ulna rather than the bones of the lower leg, ${ }^{6}$ which we define as the portion of the body delimited by the knee and ankle. Reports of bowing and pseudarthrosis of the humerus and clavicle are rare but have also been published.7,8

In addition, the term pseudarthrosis is clearly inappropriate when applied to a bone that has never fractured (or has fractured and healed adequately). Moreover, although anterolateral bowing of the lower leg is sometimes congenital and is usually recognized in infancy, fracture of a dysplastic tibia rarely occurs before birth. In addition, some individuals with unequivocal tibial dysplasia and anterolateral bowing do not fracture, and some who sustain a fracture heal adequately and do not develop pseudarthrosis. Therefore, the classic term congenital tibial pseudarthrosis used so often in the genetics and orthopedic literature is not the most appropriate term for this progressive process involving the long bones in individuals with NF1.
In 2000, the National Neurofibromatosis Foundation (now Children's Tumor Foundation) convened a task force on bone abnormalities in NF1 in Salt Lake City, UT, to discuss the natural history of long bone dysplasia and dysplastic scoliosis in NF1. Since that time, progress has been made in describing the clinical presentation, natural history, and radiographic findings of long bone bowing and pseudarthrosis in NF1.4,6,9

\section{CHARACTERISTIC CLINICAL AND RADIOGRAPHIC FINDINGS IN NF1 PATIENTS WITH LONG BONE DYSPLASIA}

Anterolateral bowing of the lower leg, with the apex of the convexity near the junction of the middle and distal thirds of the tibia is the usual presenting clinical sign in an infant or child with long bone dysplasia characteristic of NF1. In some instances, fracture or pseudarthrosis has already occurred, but this is not always the case. Of note, pseudarthrosis without 
previous bowing of the long bone should be considered unrelated to NF1 unless other diagnostic criteria are satisfied.

Although some NF1 patients with tibial dysplasia do have thinning of the cortex on radiographic examination, most do not. ${ }^{4,9}$ The most characteristic radiographic findings in a dysplastic tibia before fracture are cortical thickening with medullary canal narrowing that is greatest near the maximum point of bowing, usually located at the junction of the middle and distal thirds of the tibia (Fig. 1). Various other radiographic findings have also been observed in affected bones, including an increased width of the medullary canal with tubulation defects, cystic lesions, and a dysplastic constriction of the long bone. ${ }^{10}$ In addition, osteopenia and cortical thinning at the very proximal and distal ends of the long bone can be seen after a fracture, but these abnormalities are probably secondary osseous changes that result from disuse.

Several radiographic classification systems have been proposed for tibial dysplasia, including the classifications of Boyd, Andersen, and Crawford. ${ }^{4,10-21}$ It is, however, important to realize that $20 \%$ to $50 \%$ of individuals with tibial pseudarthrosis do not have NF1, $19,21,22$ and some of the radiographic findings described in the various classification systems may represent features of conditions other than NF1. Other etiologies that have been suggested for tibial pseudarthrosis include intrauterine trauma, rickets, osteogenesis imperfecta, amniotic bands, fibrous dysplasia, and endocrine abnormalities. ${ }^{23}$ In addition, some radiographic findings described in these classification systems may represent later stages of the disease, whereas other features may be typical of the findings at first presentation, before fracture, nonunion, or the effects of various treatments. ${ }^{9}$

Given that the radiographic findings are not static, it is important to consider what time period is most germane to the use of characteristic skeletal features as a diagnostic criterion for NF1. Cutaneous pigmentary, ocular, and tumor features of NF1 that are used as diagnostic features accumulate with age. ${ }^{3,24}$ Therefore, use of characteristic long bone dysplasia as a diagnostic criterion is most likely to be important in infancy or early childhood. At this time of life, tibial dysplasia is most likely to present as anterolateral bowing. In addition, cortical thickening rather than thinning is most likely to be observed radiographically. Regardless of the radiographic findings, clinical presentation of anterolateral bowing of the lower leg is probably adequate as a diagnostic criterion for NF1.

\section{PROPOSED CLARIFICATION OF THE DIAGNOSTIC CRITERION}

The terminology of the example of a distinctive osseous lesion pertaining to long bone dysplasia currently used in the diagnostic criteria for NF1 is misleading for clinicians and researchers as a diagnostic criterion. Notably, long bone bowing, which is not mentioned as an example in the NF1 diagnostic criterion, is the primary clinical finding in long bone dysplasia. We propose to eliminate the example of "thinning of the cortex, with or without pseudarthrosis" used in the diagnostic criterion. If one were to use the example strictly as stated, relatively few NF1 individuals presenting with long bone bowing would meet this criterion. Anterolateral bowing of the lower leg, with or without fracture or pseudarthrosis, is the primary finding a clinician should use to fulfill the sixth diagnostic criterion for NF1.

The NF1 diagnostic criteria proposed in 1987 are well entrenched in medical practice for the clinical evaluation of individuals suspected to have NF1. ${ }^{1}$ It is likely that most physicians in NF1 specialty clinics recognize the range of osseous features that are characteristic for NF1. Why is it important to clarify the diagnostic criterion related to characteristic osseous manifestations? In the absence of clinical bowing of the lower leg or leg pain, clinicians not familiar with NF1 may obtain radiographs and request cortical measurements to look for cortical thinning, although this is not clinically indicated. In addition, clinical recognition of anterolateral bowing as the cardinal sign of tibial dysplasia will facilitate early referral to an orthopedist for preventive and therapeutic strategies including bracing to avoid fracture and pseudarthrosis. ${ }^{4}$ Also, the diagnostic criteria are important for appropriate inclusion in most clinical research protocols investigating NF1.

Parents of young children with isolated café-au-lait macules who do not yet fulfill the diagnostic criteria for NF1 may also be misled by the current terminology for the sixth diagnostic criterion. This is evidenced in literature circulated by national support groups for NF1. For example, a "Questions and Answers" pamphlet published by the Children's Tumor Foundation in 2005 states that one of the findings characterizing NF1 is "thinning of the shin bone." It would be much more helpful to describe this finding as bowing of the lower leg toward the front and side of the body. Appropriate interpretation of the sixth diagnostic criterion should help to increase our understanding of the natural history and pathophysiology of NF1 and improve the clinical care of individuals with this condition.

\section{ACKNOWLEDGMENTS}

This study was carried out with support in part from a grant from the Shriners Research Foundation, a Public Health Services research grant M01-RR00064 from the National Center for Research Resources, research grants K23 NS052500 and R01 NS050509-01A1 from the National Institute of Neurological Disorders and Stroke, Department of Defense CDMRP grant NF050133, the Children's Health Research Center at the University of Utah, and the Primary Children's Research Foundation. Concepts were derived in part from a meeting of members of the International NF1 Bone Study Group (INBSG).

\section{References}

1. Neurofibromatosis. Conference statement. National Institutes of Health Consensus Development Conference. Arch Neurol 1988;45:575-578.

2. Gutmann DH, Aylsworth A, Carey JC, Korf B, et al. The diagnostic evaluation and multidisciplinary management of neurofibromatosis 1 and neurofibromatosis 2 . JAMA 1997;278:51-57.

3. Friedman JM, Birch PH. Type 1 neurofibromatosis: a descriptive analysis of the disorder in 1728 patients. Am J Med Genet 1997;70:138-143.

4. Crawford AH, Schorry EK. Neurofibromatosis in children; the role of the orthopaedist. J Am Acad Orthop Surg 1999;7:217-230. 
5. Crawford AH, Schorry EK. Neurofibromatosis update. J Pediatr Orthop 2006;26: 413-423.

6. Stevenson DA, Birch PH, Friedman JM, Viskochil DH, et al. Descriptive analysis of tibial pseudarthrosis in patients with neurofibromatosis 1. Am J Med Genet 1999;84: 413-419.

7. Moore JR. Delayed autogenous bone graft in the treatment of congenital pseudarthrosis. J Bone Joint Surg Am 1949;31:23.

8. Rudicel S. The orthopaedic manifestations of neurofibromatosis. Conn Med 1987; 51:221-222.

9. Hefti F, Bollini G, Dungl P, Fixsen J, et al. Congenital pseudarthrosis of the tibia: history, etiology, classification, and epidemiologic data. J Pediatr OrthopB 2000;9: $11-15$.

10. Crawford AH, Bagamery N. Osseous manifestations of neurofibromatosis. J Pediatr Orthop 1986;6:72-88.

11. Andersen KS. Radiological classification of congenital pseudarthrosis of the tibia. Acta Orthop Scand 1973;44:719-727.

12. Andersen KS. Congenital pseudarthrosis of the tibia and neurofibromatosis. Acta Orthop Scand 1976;47:108-111.

13. Andersen KS. Congenital pseudarthrosis of the leg. J Bone Joint Surg Am 1976;58: $657-662$.
14. Bassett CAL, Caulo N, Korte GJ. Congenital pseudarthrosis of the tibia: treatment with pulsing electromagnetic fields. Clin Orthop 1980;154:136-149.

15. Boyd HB, Sage FP. Congenital pseudarthrosis of the tibia. J Bone Joint Surg Am 1958;40:1245-1270.

16. Crawford AH. Neurofibromatosis in children. Acta Orthop Scand 1986;57:7-60.

17. Masserman RL, Peterson HA, Bianco AJ. Congenital pseudarthrosis of the tibia: a review of the literature and 52 cases from the Mayo Clinic. Clin Orthop 1974;99:140145.

18. McFarland B. Pseudarthrosis of the tibia in childhood. J Bone Joint Surg Br 1951;33: 35-46.

19. Morrissy RT, Riseborough EJ, Hall JE. Congenital pseudarthrosis of the tibia. J Bone Joint Surg Br 1981;63:367-375.

20. Rathgeb JM, Ramsey PL, Cowell HR. Congenital kyphoscoliosis of the tibia. Clin Orthop 1974;103:178-190.

21. Sofield HA. Congenital pseudarthrosis of the tibia. Clin Orthop 1971;76:33-42.

22. Gilbert A, Brockman R. Congenital pseudarthrosis of the tibia. Clin Orthop 1995; 314:37-44.

23. Moore JR. Congenital pseudarthrosis of the tibia. Instr Course Lect 1957;14:222-237.

24. Young H, Hyman S, North K. Neurofibromatosis 1: clinical review and exceptions to the rules. J Child Neurol 2002;17:613-621. 